\title{
Silk Nanoparticle Manufacture in Semi-Batch Format
}

\author{
Saphia A. L. Matthew, John D. Totten, Suttinee Phuagkhaopong, Gemma Egan, Kimia Witte, \\ Yvonne Perrie, and F. Philipp Seib*
}

Cite This: https://dx.doi.org/10.1021/acsbiomaterials.0c01028

Read Online

ACCESS | W Metrics \& More | 回 Article Recommendations

ABSTRACT: Silk nanoparticles have demonstrated utility across a range of biomedical applications, especially as drug delivery vehicles. Their fabrication by bottom-up methods such as nanoprecipitation, rather than top-down manufacture, can improve critical nanoparticle quality attributes. Here, we establish a simple semi-batch method using drop-by-drop nanoprecipitation at the lab scale that reduces special-cause variation and improves mixing efficiency. The stirring rate was an important parameter affecting nanoparticle size and yield $(400<200<0 \mathrm{rpm})$, while the initial dropping height ( 5.5 vs $7.5 \mathrm{~cm}$ ) directly affected nanoparticle yield. Varying the nanoparticle standing time in the mother liquor between 0 and $24 \mathrm{~h}$ did not significantly affect nanoparticle physicochemical properties, indicating

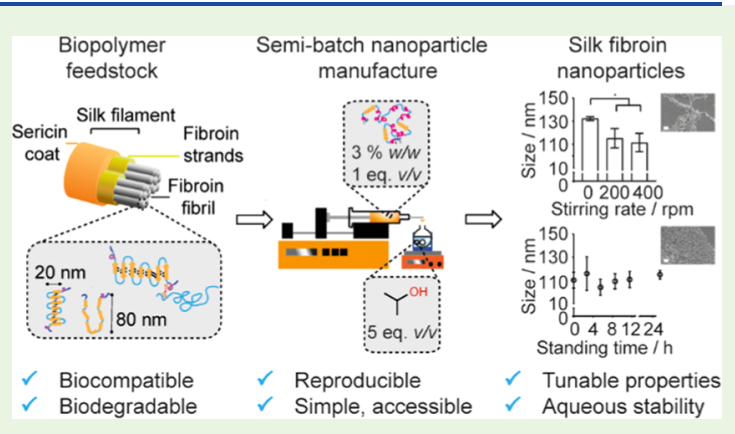
that steric and charge stabilizations result in high-energy barriers for nanoparticle growth. Manufacture across all tested formulations achieved nanoparticles between 104 and $134 \mathrm{~nm}$ in size with high $\beta$-sheet content, spherical morphology, and stability in aqueous media for over 1 month at $4{ }^{\circ} \mathrm{C}$. This semi-automated drop-by-drop, semi-batch silk desolvation offers an accessible, higherthroughput platform for standardization of parameters that are difficult to control using manual methodologies.

KEYWORDS: silk fibroin, nanoprecipitation, desolvation, nanoparticle, biopolymer

\section{INTRODUCTION}

The mulberry silk produced by the Bombyx mori silkworm is one of the most extensively studied silks, with ancient and farreaching applications ranging from domestic to medical textiles. ${ }^{1,2}$ The ability to regenerate silk fibroin protein from the silk cocoon has realized an advent of new material formats with adjustable physical properties; most notable among these formats are porous scaffolds, ${ }^{3}$ hydrogels, ${ }^{4}$ films, ${ }^{5}$ and particles. ${ }^{6}$ Silk fibroin offers several exploitable characteristics, including broad biocompatibility and biodegradability, ${ }^{5,7}$ low immunogenicity, ${ }^{5}$ and the presence of reactive amino acids amenable to chemical modification. ${ }^{8}$ This amenability makes reverseengineered silk a promising precursor for clinical applications, ${ }^{1,4}$ as evidenced by the granting in 2019 of the first FDA approval for a regenerated silk hydrogel for human vocal fold reinforcement (Silk Voice, Sofregen Medical Inc., Medford, MA). ${ }^{9}$

B. mori silk fibroin is a structural protein composed of a light $(\approx 26 \mathrm{kDa})^{1}$ and a heavy chain $(\approx 391 \mathrm{kDa}){ }^{1}$, which are linked by a disulfide bond. ${ }^{1}$ The heavy chain has a block copolymer sequence of short hydrophilic amorphous regions interspersed with long hydrophobic (GAGAGX) ${ }_{n}$ and (GAGAGY) residues. ${ }^{1}$ These hydrophobic motifs, which are capable of $\beta$ sheet self-assembly and constitute over $50 \%$ of the primary structure, impart high mechanical strength to the fiber. ${ }^{5}$ Silk is a natural biopolymer with metastable tertiary structures; therefore, the structure of silk-based materials can be tuned to their desired function by modifying their crystallinity ${ }^{1,3,4,10}$ and hierarchical composition. ${ }^{11}$

This structural versatility, coupled with the amphiphilic nature of silk, also permits silk to undergo a variety of favorable intermolecular interactions with lipophilic and hydrophilic therapeutic payloads ${ }^{3,12}$ by in situ ${ }^{12}$ or postsynthetic loading. ${ }^{12-14}$ These interactions can also stabilize synthetic drugs $^{3,12}$ and biological molecules ${ }^{3,12}$ by surface adsorption or encapsulation, thereby sterically shielding a drug cargo from biological clearance. The drug release behavior can be designed according to a tissue-specific stimulus to improve efficacy and reduce off-target effects while preserving drug structure and activity. ${ }^{3,12,15}$ Silk nanoparticles are especially suited for drug targeting of solid tumors as these nanoparticles exhibit increased drug release at low $\mathrm{pH}^{6,8}$ which is a signature of tumor environments. In addition, silk nanoparticles have shown desirable critical quality attributes, including in vitro endocytosis-mediated uptake, ${ }^{14}$ lysosomotropic drug release, ${ }^{16}$ and proteolysis, ${ }^{16,17}$ which indicate their value as anticancer nanomedicines.

Received: July 10, 2020

Accepted: October 15, 2020 


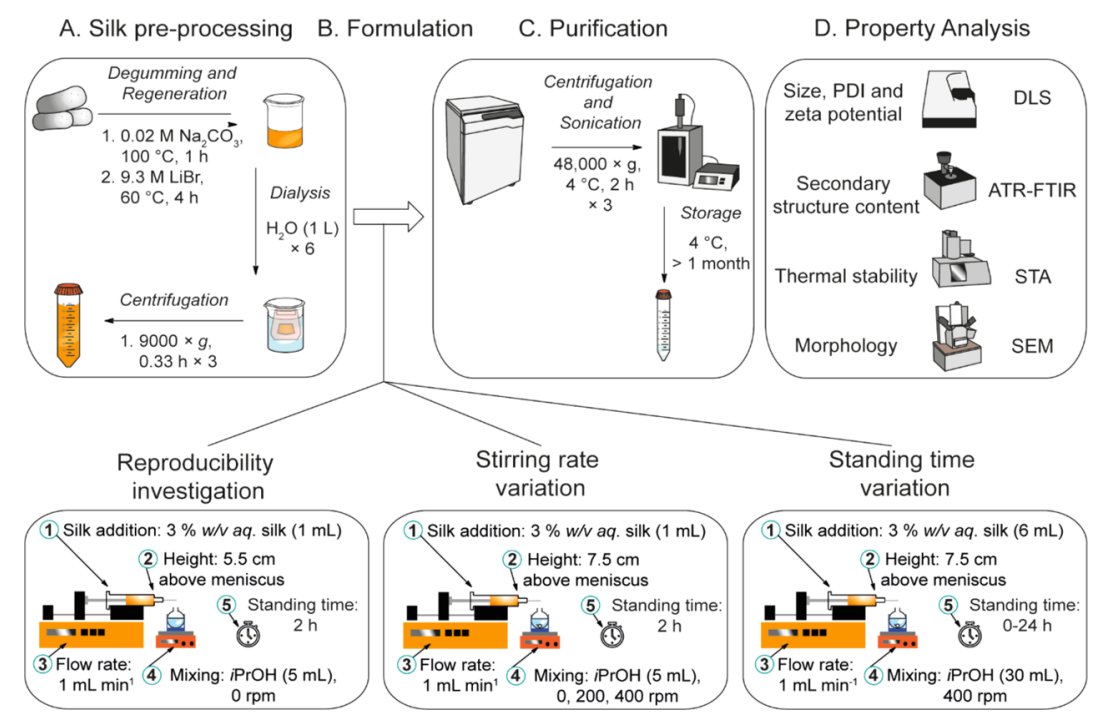

Figure 1. Nanoprecipitation workflow in a drop-by-drop open system for the preparation of silk nanoparticles. The five processing steps are (1) loading of a bubble-free $3 \% \mathrm{w} / \mathrm{v}$ aqueous silk solution into a syringe equipped with a blunt needle, (2) the relative positions of the needle and round-bottom flask, (3) the flow rate control of silk solution at $1 \mathrm{~mL} \mathrm{~min}^{-1},(4)$ the stirring rate during addition, and (5) the nanoparticle standing time in the mother liquor following completion of silk addition.

Preparation of silk particles of submicron size $(25-180 \mathrm{~nm})$ can be achieved by six major bottom-up methods (reviewed previously $\left.{ }^{18}\right)$ : capillary microdot printing, ${ }^{19}$ desolvation, ${ }^{8,13,14,20}$ supercritical $\mathrm{CO}_{2}{ }^{21}$ and electrospraying, ${ }^{22}$ emulsification, ${ }^{23}$ and ionic liquid dissolution. ${ }^{24}$ Among these methods, desolvation provides one of the most accessible and least energy-intensive lab-scale methods and is commonly used for the manufacture of protein nanoparticles. ${ }^{25}$ Desolvation of silk is a nanoprecipitation process whereby an aqueous silk solution is mixed with a water-miscible organic solvent in which the heavy-chain hydrophilic blocks have low solubility (e.g. isopropanol and acetone). This process has no requirement for method-specific, expensive apparatus ${ }^{18}$ and produces silk nanoparticles with cores enriched in $\beta$-sheet structures without the need for further chemical cross-linking steps.

Currently, optimized lab-scale desolvation methodology uses a semi-batch format consisting of a manual drop-by-drop addition of $3-5 \% \mathrm{w} / \mathrm{v}$ silk into at least a $200 \% \mathrm{v} / \mathrm{v}$ excess of the organic antisolvent. ${ }^{8,13,14}$ In comparison to batch processes, where an empty reactor is charged with all species simultaneously, semi-batch desolvation is defined by the feed of the solute into a vessel precharged with an antisolvent or vice versa. ${ }^{26}$ Semi-batch nanoprecipitation can be scaled up from the bench, ${ }^{26}$ with the process further aided by computational simulations. ${ }^{27,28}$ However, when compared to pilot-scale operations, the manual method suffers from specialcause variations in flow rate, droplet size, and dropping height. Additionally, although particle size and polydispersity are controlled by rapid mixing, ${ }^{26,27}$ which is facilitated by agitation, ${ }^{26,28}$ stirring is not a common practice in manual silk desolvation procedures.

Designing procedures, which reduce processing times and batch-to-batch variability, will aid the progress of pharmaceutical products from the bench to the market. ${ }^{4,12}$ The aim of the current study was to establish a simple, semi-automated, and higher-throughput drop-by-drop technique for semi-batch silk nanoprecipitation. We investigated the impact of several process parameters, including stirring rate and standing time, on the physicochemical properties (e.g. particle size, polydispersity, zeta potential, stability, secondary structure, morphology, and yield) of the resulting silk nanoparticles.

\section{MATERIALS AND METHODS}

Unless otherwise stated, studies were conducted at $18-22^{\circ} \mathrm{C}$. All reagents and solvents were acquired from Acros Organics or SigmaAldrich at $>98 \%$ purity, unless otherwise stated, and utilized without additional purification.

2.1. Regeneration of $B$. mori Silk. Silk fibroin was extracted from B. mori cocoons, as described elsewhere. ${ }^{13}$ Briefly, B. mori cocoons were cut into approximately $5 \times 5 \mathrm{~mm}^{2}$ sections and boiled, with manual stirring, in $0.02 \mathrm{M}$ aqueous $\mathrm{Na}_{2} \mathrm{CO}_{3}(2 \mathrm{~L})$ at $98-105^{\circ} \mathrm{C}$ for $1 \mathrm{~h}$. Degummed silk fibers were rinsed in ultrapure $\mathrm{H}_{2} \mathrm{O}(1 \mathrm{~L})$ three times for $0.33 \mathrm{~h}$ each. The silk was then dried for at least $24 \mathrm{~h}$ at room temperature.

Dry silk fibers were dissolved in a $9.3 \mathrm{M}$ aqueous $\mathrm{LiBr}$ solution at $60{ }^{\circ} \mathrm{C}$ for $4 \mathrm{~h}$ to give a $25 \% \mathrm{w} / \mathrm{v}$ silk solution. The silk solution was dialyzed (molecular weight cutoff $3500 \mathrm{~g} \mathrm{~mol}^{-1}$, Slide-A-Lyzer, Thermo Scientific, Rockford, IL) against ultrapure $\mathrm{H}_{2} \mathrm{O}(1 \mathrm{~L})$ for $48 \mathrm{~h}$ and then purified by centrifugation over four cycles, each for $0.33 \mathrm{~h}$ at $3000 \mathrm{~g}$ and $5{ }^{\circ} \mathrm{C}$ (Jouan BR4i centrifuge equipped with an S40 swing rotor). Silk concentrations were determined gravimetrically over $24 \mathrm{~h}$ at $60{ }^{\circ} \mathrm{C}$ and then adjusted to $3 \% \mathrm{w} / \mathrm{v}$ with ultrapure $\mathrm{H}_{2} \mathrm{O}$.

2.2. General Drop-By-Drop Manufacture of Silk Nanoparticles in Semi-Batch Format. Silk nanoparticles were manufactured at room temperature using a syringe pump (Harvard Apparatus 22, Holliston, MA) equipped with a BD PLASTIPACK syringe and blunt needle $\left(23 \mathrm{G} \times 0.25^{\prime \prime}\right)$ (Figure 1$)$. Inclination of the syringe pump was $0-0.1^{\circ}$. The isopropanol antisolvent was added to a short-neck round-bottom flask (to give a final 5:1 v/v ratio of isopropanol:silk). A $3 \% \mathrm{w} / \mathrm{v}$ silk solution was then added drop by drop at a rate of $1 \mathrm{~mL} \mathrm{~min} \min ^{-1}\left(\approx 27\right.$ drops $\min ^{-1}$ and $\left.37 \mu \mathrm{L} \mathrm{min}{ }^{-1}\right)$. The resulting suspensions were incubated at room temperature for the designated time and then transferred to polypropylene ultracentrifugation tubes made up to $43 \mathrm{~mL}$ with ultrapure $\mathrm{H}_{2} \mathrm{O}$ and centrifuged at $48,400 \mathrm{~g}$ for $2 \mathrm{~h}$ at $4{ }^{\circ} \mathrm{C}$ (Beckmann Coulter Avanti J-E equipped with JA- 20 rotor). The supernatant was aspirated, and the pellet was resuspended in ultrapure $\mathrm{H}_{2} \mathrm{O}(20 \mathrm{~mL})$ and sonicated twice for $30 \mathrm{~s}$ at $30 \%$ amplitude with a Sonoplus HD 2070 sonicator (ultrasonic homogenizer, Bandelin, Berlin, Germany). An additional volume of ultrapure $\mathrm{H}_{2} \mathrm{O}(23 \mathrm{~mL})$ was added, and the centrifugation, washing, and resuspension steps were repeated twice more. The final pellet was collected and resuspended in $2-3 \mathrm{~mL}$ water. This final silk 
nanoparticle suspension was stored at $4{ }^{\circ} \mathrm{C}$ until use. Unless stated otherwise, each experiment was repeated in triplicate using three different aqueous silk precursor stock solutions.

Calculations for needle residence time and shear rate are based on the literature dynamic viscosity $(27 \mathrm{mPa} \mathrm{s})$ of the regenerated $3 \%$ aqueous silk ${ }^{29}$ and density $\left(1.02 \mathrm{~g} \mathrm{~mL}^{-1}\right)$ calculated herein for the $3 \%$ $\mathrm{w} / \mathrm{v}$ aqueous silk solution and assumed Newtonian flow. ${ }^{29}$ Reynold's number was estimated as 2 using the internal diameter of the needle ${ }^{30}$ $(0.33 \mathrm{~mm})$ and indicated laminar flow. An upper limit of the residence time was estimated using the linear velocity $\left(1.94 \mathrm{~mm} \mathrm{~s}^{-1}\right)$ and the needle length. ${ }^{31}$ The maximum shear rate was taken as the wall shear rate, and for simplicity, the shear rate calculations used the geometry of a straight cylinder. Calculations for the 3 and $10 \mathrm{~mL}$ syringes used in the study were undertaken similarly using the internal diameters as stated by the manufacturer.

2.2.1. Reproducibility of Semi-Automated Silk Nanoparticle Manufacture. Silk nanoparticles were manufactured in a $10 \mathrm{~mL}$ flask at a $6 \mathrm{~mL}$ total volume (Figure 1). Silk was added from a height of 5.5 $\mathrm{cm}$ from the bottom of the isopropanol meniscus. The mother liquor suspension was then incubated for $2 \mathrm{~h}$ before purification. This procedure was repeated a further 15 times using five silk precursor solutions.

2.2.2. Effect of Stirring Rate on Manufacture and Silk Nanoparticle Properties. Silk nanoparticles were manufactured in a $10 \mathrm{~mL}$ flask at a $6 \mathrm{~mL}$ total volume (Figure 1). Silk was added from a height of $7.5 \mathrm{~cm}$ from the bottom of the isopropanol meniscus, and stirring was accomplished with an egg-shaped stir bar $(15 \times 6 \mathrm{~mm})$ at 200 and $400 \mathrm{rpm}$. The mother liquor suspension was then incubated for $2 \mathrm{~h}$ before purification. This procedure was repeated in triplicate using three silk precursor solutions.

2.2.3. Effect of Standing Time on Manufacture and Silk Nanoparticle Properties. Silk nanoparticles were manufactured in a $50 \mathrm{~mL}$ flask at a $36 \mathrm{~mL}$ total volume. Silk was added from a height of $7.5 \mathrm{~cm}$ from the bottom of the isopropanol meniscus with stirring at $400 \mathrm{rpm}$ with an egg-shaped stir bar $(15 \times 6 \mathrm{~mm})$. An aliquot $(6 \mathrm{~mL})$ was taken immediately following complete addition of the silk precursor, and stirring was stopped. Further aliquots $(6 \mathrm{~mL})$ were taken at 2.7, 5.5, 8.5, 11.5, and $24 \mathrm{~h}$ following stirring for $0.02 \mathrm{~h}$ at 400 rpm to ensure suspension homogeneity. This procedure was repeated in triplicate using three silk precursor solutions.

2.3. Yield of Silk Nanoparticles. The nanoparticle concentrations were determined by recording the total mass of the suspension in a preweighed centrifuge tube. A known mass of each suspension was then frozen at $-80{ }^{\circ} \mathrm{C}$ for $5 \mathrm{~h}$ in preweighed microcentrifuge tubes, followed by freeze-drying (Christ Epsilon 1-4, Martin Christ Gefriertrocknungsanlagen $\mathrm{GmbH}$, Osterode, Germany) for $24 \mathrm{~h}$ at $-10{ }^{\circ} \mathrm{C}$ and 0.14 mbar. The dry mass was recorded, and the yield was calculated using eq 1

$$
\text { yield } / \%=\frac{\text { particle concentration }(\% \mathrm{w} / \mathrm{w}) \times \text { mass }(\mathrm{mg})}{\text { silk concentration }(\% \mathrm{w} / \mathrm{v}) \times \text { volume }(\mathrm{mL})}
$$

$$
\times 100 \%
$$

This process was repeated in duplicate and the average yield was reported, and the freeze-dried samples were stored in a vacuum desiccator until use.

2.4. Silk Nanoparticle Physicochemical Characterization and Stability in Water. The size ( $Z$-average of the hydrodynamic diameter), polydispersity, and zeta potential of silk nanoparticles were determined as described elsewhere. ${ }^{32}$ Briefly, silk nanoparticles were analyzed in ultrapure $\mathrm{H}_{2} \mathrm{O}$ at $25^{\circ} \mathrm{C}$ by dynamic light scattering (DLS) (Zetasizer Nano-ZS Malvern Instrument, Worcestershire, U.K.). Unless otherwise stated, the samples were vortexed for $20 \mathrm{~s}$ and sonicated twice at $30 \%$ amplitude for $30 \mathrm{~s}$ prior to measurement. Refractive indices of 1.33 and 1.60 for $\mathrm{H}_{2} \mathrm{O}$ and protein, respectively, were used for particle size measurement. All analyses were conducted in triplicate.

The particle size and zeta potential of silk nanoparticles generated in the stirring studies were determined on days $0,10,18,24,28,35$, and 42 by DLS. The particle size and zeta potential of silk nanoparticles generated in standing time studies were determined at days 0,42 , and 63 by DLS. The silk nanoparticles from all studies were stored at $4{ }^{\circ} \mathrm{C}$. At $t>0$ days, the silk nanoparticles were vortexed for $20 \mathrm{~s}$ before size and zeta potential analysis.

2.5. Secondary Structure Measurements of Silk Nanoparticles. Air-dried silk films and freeze-dried silk were used as silk I structure references, while autoclaved silk films and silk films treated with $70 \% \mathrm{v} / \mathrm{v}$ ethanol/ultrapure $\mathrm{H}_{2} \mathrm{O}$ were used as positive controls for silk II structure. Silk films, powders, and nanoparticles were analyzed by Fourier transform infrared spectroscopy (FTIR) on an ATR-equipped TENSOR II FTIR spectrometer (Bruker Optik $\mathrm{GmbH}$, Ettlingen, Germany). Each nanoparticle and freeze-dried silk sample was flash-frozen at $-80{ }^{\circ} \mathrm{C}$ for at least $5 \mathrm{~h}$ and then lyophilized for $24 \mathrm{~h}$. Each FTIR measurement was run for 128 scans at a $4 \mathrm{~cm}^{-1}$ resolution in absorption mode over the wavenumber range of $400-4000 \mathrm{~cm}^{-1}$ and corrected for atmospheric absorption using Opus (Bruker Optik GmbH, Ettlingen, Germany). The amide I regions of the FTIR spectra were analyzed in OriginLab 19b (Northampton, Massachusetts), as described elsewhere. ${ }^{33}$ The second derivative of the background-corrected absorption spectrum was obtained and smoothed twice using a seven-point Savitzky-Golay function with a polynomial order of 2 . A nonzero linear baseline was interpolated between 2 and 3 of the highest points between 1600 and $1710 \mathrm{~cm}^{-1}$. Peak positions in the amide I region were then identified using the second derivative and peaks fitted using nonlinear least squares with a series of Gaussian curves (Figure S1). Band positions, widths, and heights were allowed to vary, and peak area was allowed to take any value below or equal to 0 . The deconvoluted spectra were area-normalized, and the secondary structure content was calculated with reference to literature band assignments ${ }^{34,35}$ using the relative areas of each band.

The correlation coefficient $(R)$ was calculated according to previous analyses. ${ }^{36}$ The air-dried silk film of an aqueous silk precursor batch was used as the reference for all silk films, freeze-dried silk, and silk nanoparticle samples. The second-derivative curves of the absorption spectra were smoothed twice with a five-point Savitzky-Golay function and a polynomial order of 2 and then compared over the spectral range of $1600-1700 \mathrm{~cm}^{-1}$ using eq 2

$$
R=\frac{\sum x_{\mathrm{i}} y_{\mathrm{i}}}{\sqrt{\sum x_{\mathrm{i}}^{2} \sum y_{\mathrm{i}}^{2}}}
$$

where $x_{i}$ and $y_{i}$ are the derivative values of the air-dried silk film and sample of interest at the frequency $i$, respectively.

2.6. Thermal Analysis of Silk Nanoparticles. A known volume and mass of each silk sample and freeze-dried silk control was frozen at $-80^{\circ} \mathrm{C}$ for $5 \mathrm{~h}$, followed by freeze-drying for $24 \mathrm{~h}$ at $-10{ }^{\circ} \mathrm{C}$ and 0.14 mbar. First-cycle differential scanning calorimetry and thermogravimetric analysis were carried out on the dried samples $(1.95-4.89 \mathrm{mg})$ in aluminum pans from 20 to $350{ }^{\circ} \mathrm{C}$ at a scanning

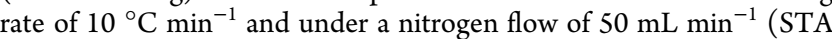
Jupiter 449, Netzsch, Gerätebau GmbH, Germany). Thermograms were analyzed using OriginLab 19b (Northampton, Massachusetts). The desorption enthalpy was normalized to a corrected mass during volatilization, as described previously. ${ }^{37}$

2.7. Scanning Electron Microscopy (SEM) of Silk Nanoparticles. Aqueous silk nanoparticle suspensions were adjusted to a concentration of $1 \mathrm{mg} \mathrm{mL}^{-1}$. An aliquot $(20 \mu \mathrm{L})$ of each sample was then pipetted onto a silicon wafer and lyophilized for $24 \mathrm{~h}$ at $-10{ }^{\circ} \mathrm{C}$ and 0.14 mbar. The specimens were sputter-coated with gold using a low-vacuum sputter coater (Agar Scientific Ltd., Essex, U.K.) and analyzed with the secondary electron detector of an FE-SEM SU6600 instrument (Hitachi High Technologies, Krefeld, Germany) at $5 \mathrm{kV}$ and $40 \mathrm{k}$ magnification. The images were processed using ImageJ v1.52n (National Institutes of Health, Bethesda, MD) and Adobe Illustrator (Adobe, San Jose, CA).

2.8. Statistical Analyses. Data were analyzed using Microsoft Excel 2019 (Microsoft Office 365 ProPlus Software, Redmond, WA), Minitab (Minitab Statistical Software, State College, PA), and GraphPad Prism 8.2.1 (GraphPad Software, La Jolla, CA). The test 
for equal variance was undertaken on multiple groups using Bartlett's method. Sample pairs were analyzed using Welch's independent $t$-test. Multiple groups were evaluated by one-way analysis of variance (ANOVA), followed by Tukey's multiple comparison post hoc test or by the Brown-Forsythe and Welch ANOVA tests, followed by the Dunnett T3 multiple comparison post hoc test. Silk nanoparticle stability was evaluated by ANOVA followed by Dunnett's post hoc test to compare between $t=0$ day control and $t>0$ day samples. All data were assumed to have normal distributions. Asterisks denote statistical significance determined using post hoc tests as follows: $* p<$ $0.05, * * p<0.01$, ***p $<0.001$, and $* * * * p<0.0001$. Unless otherwise specified, all data are presented as mean values $\pm(\mathrm{SD})$ and the number of experimental repeats $(n)$ is noted in each figure legend.

\section{RESULTS}

3.1. Silk Nanoparticle Characterization. The DLS and mass measurement values indicated an influence of the stirring rate on the physicochemical properties and yield of silk nanoparticles (Figures 1 and 2). When the silk solution was
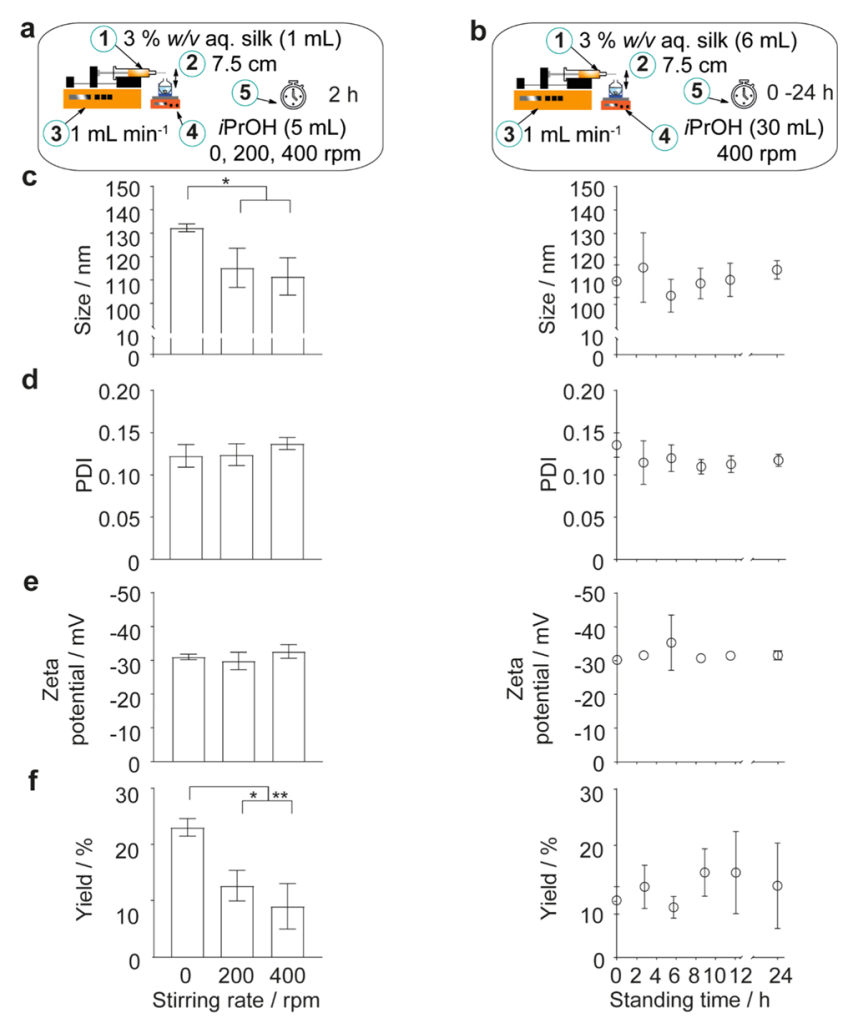

Figure 2. Impact of stirring rate and standing time on the physicochemical properties and yield of nanoparticles produced by drop-by-drop desolvation. (a) Schematic of the five processing parameters used to investigate the stirring rate and (b) the standing time for silk nanoparticle manufacture. (c) Hydrodynamic diameter, (d) polydispersity index (PDI), (e) zeta potential, and (f) yield of silk nanoparticles. Error bars are hidden in the bars and plot symbols when not visible, $\pm S D, n=3$. Multiple groups were evaluated by oneway analysis of variance (ANOVA), followed by Tukey's multiple comparison post hoc test. Asterisks denote statistical significance determined using post hoc tests as follows: $*_{p}<0.05$ and ${ }^{*} p<0.01$.

added from a height of $7.5 \mathrm{~cm}$, an increase in the stirring rate from 0 to $400 \mathrm{rpm}$ significantly decreased the silk nanoparticle size (ANOVA, $p<0.05$ ) from 134 to $114 \mathrm{~nm}$ (Figure 2c). Varying the stirring rate between 0 and $400 \mathrm{rpm}$ had no significant impact on the polydispersity or negative surface charge, which ranged from 0.12 to 0.14 and -30 to $-33 \mathrm{mV}$, respectively (Figure 2d,e). However, increasing the stirring rate significantly decreased the yield from 23 to $9 \%$ (ANOVA, $p<$ 0.01) (Figure 2f).

The effect of droplet velocity on nanoparticle formation in the absence of stirring was determined by varying the height from which the silk solution was dropped (henceforth, initial addition height). A decrease in the initial addition height from $7.5 \mathrm{~cm}\left(\nu_{\text {droplet }} \approx 1.21 \mathrm{~m} \mathrm{~s}^{-1}\right)$ to $5.5 \mathrm{~cm}\left(\nu_{\text {droplet }} \approx 1.03 \mathrm{~m} \mathrm{~s}^{-1}\right)$ significantly decreased the yield of nanoparticles ( $t$-test, $p<$ 0.01 ) from 23 to $18 \%$. By contrast, the physicochemical properties were not affected by decreasing the initial addition height to $5.5 \mathrm{~cm}$, as the nanoparticles had an average size, polydispersity, and zeta potential of $131 \mathrm{~nm}, 0.11$, and -30 $\mathrm{mV}$, respectively.

The growth of nanoparticles in the mother liquor was also investigated by varying the nanoparticle standing time before purification (Figure 1). Over a $24 \mathrm{~h}$ interval, the standing time had no significant effect on nanoparticle physicochemical properties or yield. Overall, the silk nanoparticle size ranged from 104 to $116 \mathrm{~nm}$, with a polydispersity ranging from 0.11 to 0.14 . The negative surface charge ranged from -30 to -35 $\mathrm{mV}$, and the yield varied between 9 and $15 \% \mathrm{w} / \mathrm{w}$ of silk (Figure 2).

3.2. Secondary Structure Measurement. The impact of the process conditions on silk nanoparticle secondary structure was determined by attenuated total reflectance-FTIR (ATRFTIR) analysis and deconvolution of the characteristic protein amide I band (1600-1710 $\mathrm{cm}^{-1}$ ) (Figure S1). Silk nanoparticle secondary structure did not vary significantly with changes in the initial addition height, stirring rate, or formulation standing time. For stirring and standing time studies, the high nanoparticle $\beta$-sheet content (54-57\%) correlated with the $55 \% \beta$-sheet composition measured for autoclaved and ethanol-treated silk films, which served as positive controls for silk II structure (Figure 3). Additionally, the $\alpha$-helix and random coil content $(18-21 \%)$ of silk nanoparticles were comparable to autoclaved (20\%) and ethanol-treated films (19\%). Autoclaving provides thermal energy to break labile bonds in the silk film, with the uptake of water acting to plasticize the material. This directly contrasts with nanoprecipitation, where water is removed from the silk hydration shell. The silk nanoparticle structure from both studies showed a significantly higher percentage of $\beta$-sheets (ANOVA, $p<0.0001$ ) and less $\alpha$-helix and random coil content (ANOVA, $p<0.0001)$ compared to the negative silk II structure controls (air-dried silk film and freeze-dried silk powder with $17-25 \% \quad \beta$-sheet and $47-56 \% \alpha$-helix and random coil content) (Figure $3 \mathrm{c}$ ).

The spectral correlation coefficient method of comparing second-derivative ATR-FTIR spectra in the amide I region (1600-1700 $\mathrm{cm}^{-1}$ ) was also used to measure formulationinduced structural changes in silk nanoparticles versus those in an air-dried silk film. Silk nanoparticle correlation coefficients ranged from 0.27 to 0.31 and showed no significant variation with initial addition height, stirring rate, or formulation standing time (Figure 3c). The stirring and standing time studies revealed a discrepancy between the correlation coefficients of silk nanoparticles and those of the autoclaved films (0.10) (ANOVA, $p<0.0001)$ and the ethanol-treated silk films $(0.18)$ (ANOVA, $p<0.05)$. This disagreement with the band deconvolution findings could reflect offsets in the secondderivative baselines. Regardless, the nanoprecipitation-associated $\beta$-sheet enrichment, identified by band deconvolution, 
a

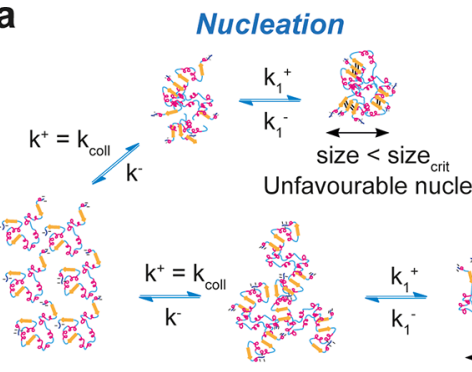

Diffusion-limited and polynuclear surface-controlled growth

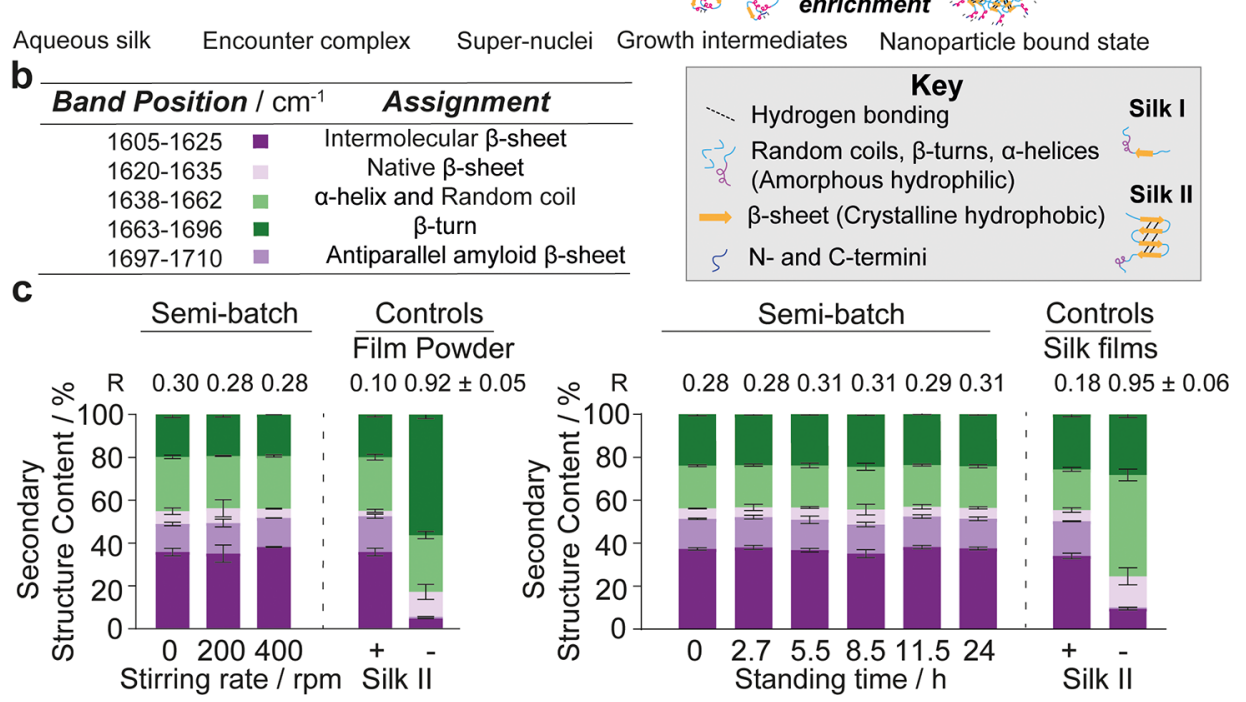

Figure 3. Changes in the stirring rate or standing time results in silk nanoparticles with comparable secondary structure. (a) Schematic of proteinprotein association and $\beta$-sheet assembly of silk fibroin during nanoprecipitation via desolvation. Silk molecules, nanoparticles, and stoichiometry of association are not drawn to scale. (b) FTIR band assignments and schematic key. Secondary structure content of silk nanoparticles manufactured using different $(\mathrm{c})$ stirring rates and (d) standing times. Silk films treated with $70 \%$ ethanol and autoclaving to obtain high $\beta$-sheet content were used as positive controls for silk II structure, with an untreated silk film and freeze-dried silk powder serving as negative controls. The correlation coefficients $(R)$ of silk nanoparticle, film, and powder second-derivative amide I spectra were calculated using the silk II negative control film as reference, $n=3, \pm \mathrm{SD}$. The correlation coefficients, total $\beta$-sheet, and $\alpha$-helix and random coil contents were evaluated by one-way analysis of variance (ANOVA), followed by Tukey's multiple comparison post hoc test. The intermolecular $\beta$-sheet, native $\beta$-sheet, $\beta$-turn, and antiparallel amyloid $\beta$-sheet contents were evaluated using the Brown-Forsythe and Welch ANOVA tests, followed by the Dunnett T3 multiple comparison post hoc test.

was supported by the significantly lower nanoparticle correlation coefficients compared to the negative silk II controls of air-dried (0.95) and freeze-dried (0.92) silks (ANOVA, $p<0.0001$ ).

3.3. Thermal Analysis. The simultaneous thermal analysis first-cycle results of silk nanoparticles manufactured at stirring rates between 0 and $400 \mathrm{rpm}$ and silk II negative controls are shown in Figure 4 and Table 1. Thermogravimetric analysis (TGA) was used to confirm differences in water content and thermal stabilities of silk nanoparticles caused by formulation (Figure 4). Thermograms of all silk nanoparticles and controls showed three regions with two weight loss steps: the loss of adsorbed and strongly bound water between 20 and $140{ }^{\circ} \mathrm{C}$ followed by silk decomposition above $170{ }^{\circ} \mathrm{C}$. The increase in mass at low temperatures in TGA measurements was due to buoyancy effects resulting from variations in air density with heating. ${ }^{38}$ No significant differences in water content were observed with increased stirring rate, with nanoparticles containing $12-14 \% \mathrm{w} / \mathrm{w}$ water across all formulations. Nanoparticles displayed a significantly delayed (ANOVA, $p<$ 0.05 ) onset decomposition temperature ranging between 273.2 and $277.3{ }^{\circ} \mathrm{C}$ compared to the freeze-dried powder, negative silk II control $\left(261.4{ }^{\circ} \mathrm{C}\right)$. This higher stability to thermal degradation suggests that nanoparticle structure is composed of a higher crystalline fraction compared to amorphous, freezedried silk. Nevertheless, there was no significant difference between the decomposition temperatures of silk nanoparticles manufactured at different stirring rates, ranging between 298.5 and $304.0^{\circ} \mathrm{C}$.

Differential scanning calorimetry (DSC) measurements confirmed that the formulation stirring rate did not affect the primary or secondary structure of silk nanoparticles (Figure 4). The desorption enthalpy ranged between -207.8 and $-282.7 \mathrm{~J}$ $\mathrm{g}^{-1}$, and the temperature of desorption ranged from 36.1 to $43.6{ }^{\circ} \mathrm{C}$, with no significant variation observed with stirring rate. The water desorption-associated and final glass transitions at 59.3 and $201.5{ }^{\circ} \mathrm{C}$, respectively, were not identifiable for all nanoparticle samples. The glass transition at $201.5^{\circ} \mathrm{C}$ was also shifted to a higher temperature and was less steep when compared to that of the silk I structure $\left(184.5^{\circ} \mathrm{C}\right)$. This indicates that the molecular mobility of silk molecules was reduced upon their incorporation into the nanoparticle structure. The crystallization exotherm (random coil to $\beta$ sheet transition), present for the negative controls at $241.0^{\circ} \mathrm{C}$, was absent from the nanoparticle curves. No significant difference was noted between the decomposition temperatures 
a

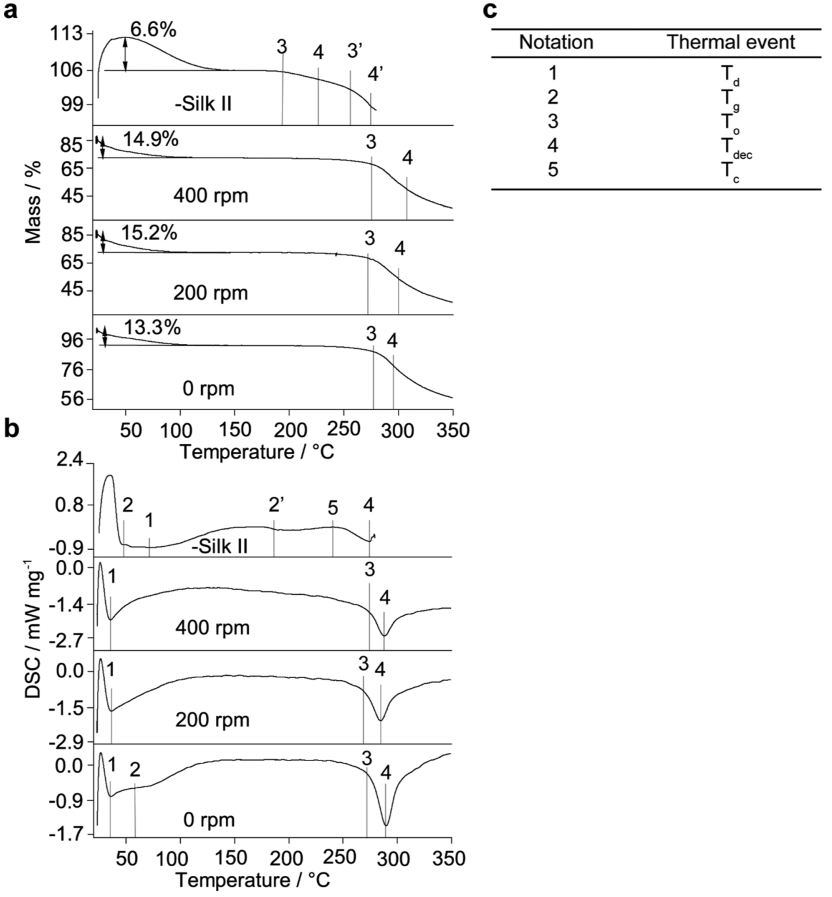

Figure 4. Representative first-cycle raw (a) thermogravimetric analysis (TGA) thermograms and (b) differential scanning calorimetry (DSC) of silk nanoparticles manufactured at different stirring rates. (c) Thermal event assignment key. Water content (\%, w/w), dehydration temperature $\left(T_{\mathrm{d}}\right)$, glass-transition temperatures $\left(T_{\mathrm{g}}\right)$, extrapolated onset temperature of crystallization and decomposition $\left(T_{\mathrm{o}}\right)$, crystallization temperature $\left(T_{\mathrm{c}}\right)$, and decomposition temperatures $\left(T_{\mathrm{dec}}\right)$ are reported.

(ranging between 282.9 and $289.5{ }^{\circ} \mathrm{C}$ ) of silk nanoparticles manufactured at different stirring rates.

3.4. Silk Nanoparticle Aqueous Stability. The particle size, polydispersity, and zeta potential stability of nanoparticles manufactured with stirring rates between 0 and $400 \mathrm{rpm}$ were determined for up to 42 days. Nanoparticles manufactured across all stirring rates showed size stability and constant polydispersity in water for up to 42 days (Figure 5). By contrast, the zeta potential of nanoparticles manufactured

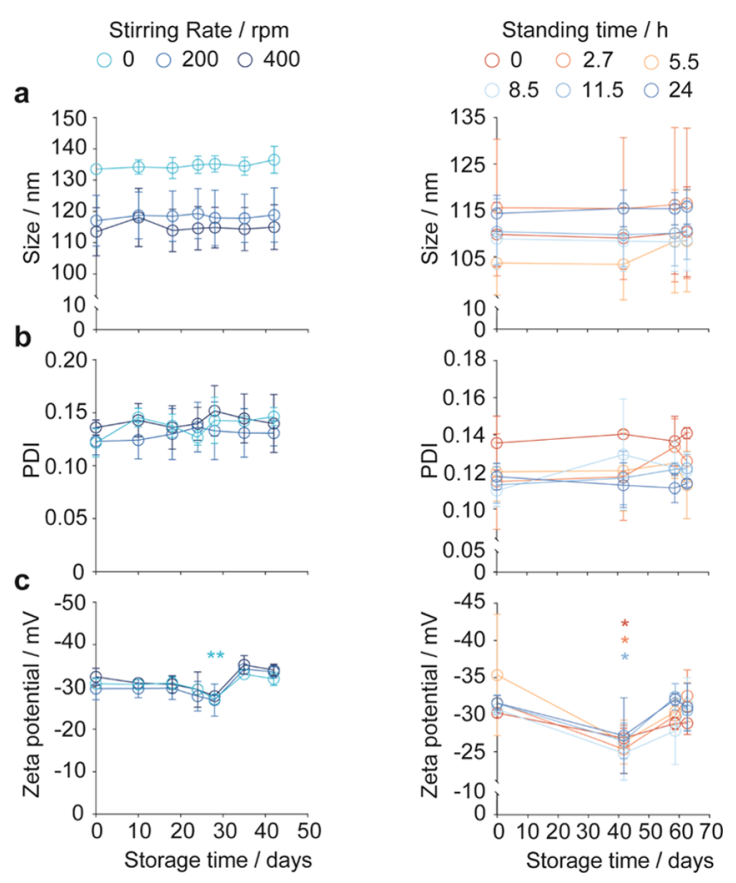

Figure 5. Stability of silk nanoparticles manufactured in semi-batch format by varying the stirring rates and standing times. (a) Hydrodynamic diameter, (b) polydispersity index (PDI), and (c) zeta potential of silk nanoparticles stored in water at $4{ }^{\circ} \mathrm{C}$. Diluted nanoparticle suspensions were vortex-mixed prior to DLS analysis, \pm $\mathrm{SD}, n=3$. Unless otherwise stated, silk nanoparticle stability was evaluated by ANOVA followed by Dunnett's post hoc test to compare between $t=0$ day control and $t>0$ day samples. The PDI stability of nanoparticles manufactured with $0 \mathrm{~h}$ standing times was evaluated using the Brown-Forsythe and Welch ANOVA followed by Dunnett's T3 post hoc test. Asterisks denote statistical significance for each formulation between $t=0$ and $t>0$ days, determined using post hoc tests as follows: ${ }^{*} p<0.05$ and $* * p<0.01$.

without stirring varied significantly across 42 days. The particle size, polydispersity, and zeta potential stability of nanoparticles manufactured with standing times between 0 and $24 \mathrm{~h}$ were also determined for up to 63 days. All formulations showed size and polydispersity stability in water for up to 63 days (Figure 5). The negative surface charges of silk nanoparticles

Table 1. First-Cycle Simultaneous Thermal Analysis Data of Silk Nanoparticles Manufactured at Different Stirring Rates

\begin{tabular}{|c|c|c|c|c|c|}
\hline & \multirow[b]{2}{*}{ thermal property } & \multicolumn{3}{|c|}{ stirring rate/rpm } & \multirow{2}{*}{$\frac{\text { silk II }}{\text { freeze-dried silk }}$} \\
\hline & & 0 & 200 & 400 & \\
\hline \multirow[t]{9}{*}{ DSC } & $T_{\mathrm{g}} /{ }^{\circ} \mathrm{C}$ & $59.3 \pm 0.01$ & $59.4^{a}$ & $59.3^{a}$ & $47.7 \pm 0.5$ \\
\hline & $T_{\mathrm{d}}(\mathrm{DSC}) /{ }^{\circ} \mathrm{C}$ & $39.1 \pm 5.3$ & $36.1 \pm 0.3$ & $43.6 \pm 13.9$ & $60.7 \pm 8.8$ \\
\hline & $\Delta H_{\mathrm{d}} / \mathrm{J} \mathrm{g}^{-1}$ & $-207.8 \pm 98.0$ & $-266.4 \pm 14.3$ & $-239.4 \pm 18.8$ & $-276.9 \pm 4.21$ \\
\hline & $T_{\mathrm{g}}{ }^{\prime} /{ }^{\circ} \mathrm{C}$ & & $196.9^{a}$ & $206.0^{a}$ & $184.5 \pm 0.7$ \\
\hline & $T_{\mathrm{o}}($ crystallization $) /{ }^{\circ} \mathrm{C}$ & & & & $225.6 \pm 11.1$ \\
\hline & $T_{\mathrm{c}} /{ }^{\circ} \mathrm{C}$ & & & & $241.0 \pm 0.8$ \\
\hline & $\Delta H_{\mathrm{c}} / \mathrm{J} \mathrm{g}^{-1}$ & & & & $9.9 \pm 2.3$ \\
\hline & $T_{\mathrm{o}} /{ }^{\circ} \mathrm{C}$ & $274.0 \pm 0.3$ & $266.6 \pm 7.3$ & $268.9 \pm 9.0$ & \\
\hline & $T_{\mathrm{dec}} /{ }^{\circ} \mathrm{C}$ & $289.5 \pm 0.5$ & $282.9 \pm 6.1$ & $284.2 \pm 7.7$ & $274.9 \pm 0.7^{b}$ \\
\hline \multirow[t]{5}{*}{ TGA } & water content $/ \%$ & $13.0 \pm 1.7$ & $13.7 \pm 2.1$ & $12.6 \pm 2.0$ & $5.8 \pm 0.8$ \\
\hline & $T_{\mathrm{o}} /{ }^{\circ} \mathrm{C}$ & $277.3 \pm 0.2$ & $273.2 \pm 2.9$ & $274.3 \pm 3.8$ & $198.5 \pm 2.2$ \\
\hline & $T_{\mathrm{o}}{ }^{\prime} /{ }^{\circ} \mathrm{C}$ & & & & $261.4 \pm 2.0$ \\
\hline & $T_{\mathrm{dec}} /{ }^{\circ} \mathrm{C}$ & $299.6 \pm 6.6$ & $298.5 \pm 9.0$ & $304.0 \pm 4.6$ & $222.3 \pm 13.3$ \\
\hline & $T_{\mathrm{dec}}{ }^{\prime} /{ }^{\circ} \mathrm{C}$ & & & & $275.0 \pm 2.7$ \\
\hline
\end{tabular}

$a_{n}=1 .{ }^{b}$ An altered decomposition profile was noted for one control sample. 
a

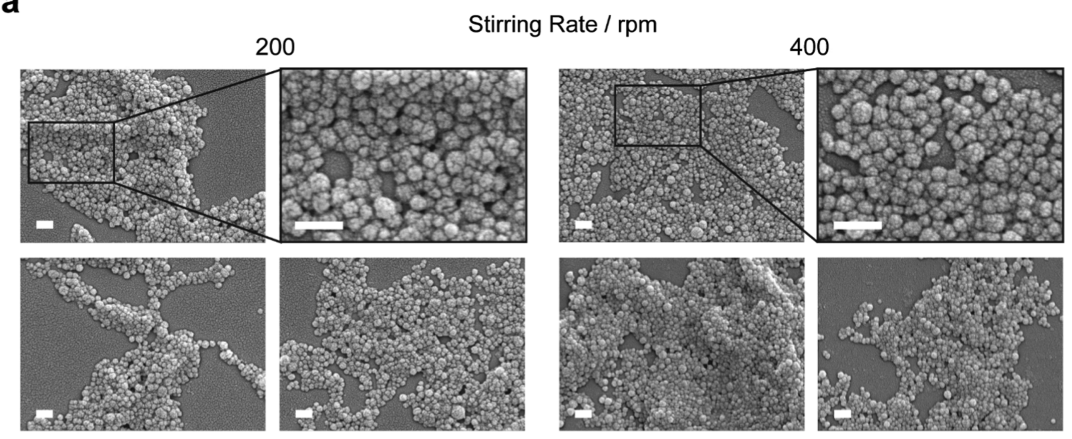

b

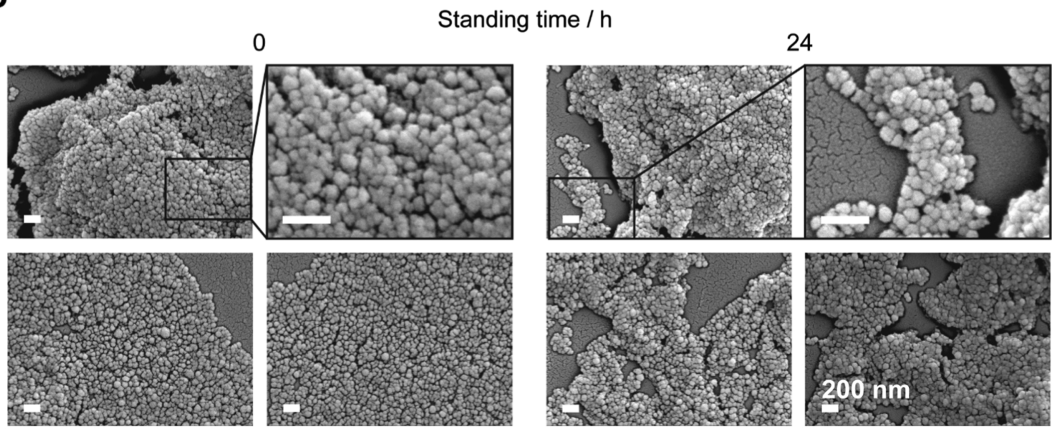

Figure 6. Scanning electron microscopy images of silk nanoparticles manufactured using (a) stirring rates of 200 and $400 \mathrm{rpm}$ and (b) standing times of 0 and $24 \mathrm{~h}$. Nanoparticles manufactured at stirring rates of 200 and $400 \mathrm{rpm}$ were imaged on day $24, n=1$. Nanoparticles manufactured using standing times of 0 and $24 \mathrm{~h}$ were imaged at day 55, $n=1$. Scale bars $=200 \mathrm{~nm}$.

after $0,2.7$, and $11.5 \mathrm{~h}$ standing times significantly decreased at 42 days at $4{ }^{\circ} \mathrm{C}$ and then increased.

3.5. Scanning Electron Microscopy of Silk Nanoparticles. Silk nanoparticle morphology was analyzed qualitatively by SEM (Figure 6). Silk nanoparticles manufactured with stirring rates of 200 and $400 \mathrm{rpm}$ at $6 \mathrm{~mL}$ scale had spherical shapes and narrow size distributions at day 24 . Nanoparticles manufactured with standing times of 0 and $24 \mathrm{~h}$ at the $36 \mathrm{~mL}$ scale showed generally spherical morphologies and uniform size distribution when imaged at day 55. Overall, nanoparticles showed a coarse surface topography.

\section{DISCUSSION}

Silk particles have attracted increased attention for drug delivery applications because their manufacture can be tailored for a desired size (from nano- to microscale), crystallinity, and surface chemistry. ${ }^{6,18,39}$ The nanoparticles produced by desolvation are well suited to anticancer applications due to their submicron size, ${ }^{8,14,16,20,32}$ which would allow extravasation through leaky tumor vasculature, ${ }^{40}$ followed by endocytosis and lysosomal trafficking in malignant cells. ${ }^{16}$ However, nanoparticle manufacture and drug loading have not always translated from small-scale, bench procedures to those following current good manufacturing practices (i.e. 21 Code of Federal Regulations Part 210-212) in the manufacturing sector. $^{41,42}$ This has prompted the implementation of continuous techniques, ${ }^{20,23,32}$ which can offer greater ease of scale-up. ${ }^{41}$ One powerful approach includes microfluidicassisted nanoprecipitation, which uses laminar flow focusing to achieve micromixing conditions and solvent shifting by diffusion. ${ }^{20,32,41}$ Production can be scaled up by microfluidicchip parallelization or increasing channel diameters, although working at large total volumes can cause problems due to low production rates and the limitations of scaling imposed by complex mixer designs. Consequently, improving the reproducibility of lab-scale methodology for semi-batch manufacture of silk nanoparticles is still an area of much interest.

Understanding the parameters that impact silk nanoprecipitation will aid in the optimization of silk nanoparticle physicochemical properties for in vivo performance as nanomedicines. The consequences of varying silk stock reverseengineering processes ${ }^{20}$ and antisolvent species, ${ }^{4,32}$ and their relative ratios, on the outcome of nanoprecipitation in semibatch $^{43}$ and continuous format ${ }^{32}$ are already reported. For example, $1 \mathrm{~h}$ degumming times for silk cocoons resulted in greater molecular weight polydispersity of silk stocks and in favorable nanoparticle size, polydispersity, and zeta potential when compared to shorter degumming times. ${ }^{20}$ Several research groups have also investigated the effect of the antisolvent species used for nanoprecipitation on the resulting nanoparticle properties, ${ }^{32,44}$ as the antisolvent molecular and macroscopic properties contribute to the mixing conditions. Increasing the volume ratio of the antisolvent-to-silk solution imposes high supersaturation conditions in both semibatch $^{43,44}$ and microfluidic formats, ${ }^{32}$ resulting in faster nucleation and smaller nanoparticle sizes. Hence, in the current study, the optimized formulation variables ${ }^{13,20,32}$ for preparing silk nanoparticles were used to investigate several little-understood preparation parameters, namely, initial addition height, nanoparticle stirring rate, and standing time.

A semi-automated drop-by-drop procedure for silk desolvation in semi-batch format was designed to replace the manual addition of silk to organic antisolvent using a syringe or pipette. It is probable that homogeneous silk nucleation occurred by antisolvent-induced desolvation (Figure 3a). We do not consider seeded crystallization as the $3 \% \mathrm{w} / \mathrm{v}$ silk concentration used lay below the $\approx 10 \% \mathrm{w} / \mathrm{w}$ critical micelle concentration $^{29}$ of regenerated silk fibroin. Additionally, the 
silk addition rate of $1 \mathrm{~mL} \mathrm{~min}{ }^{-1}$ ensured laminar flow in the syringes and needle. The maximum wall shear rates in the syringes were estimated between 55 and $261 \mathrm{~m} \mathrm{~s}^{-1}$, while the wall shear rate in the needle was estimated as $4724 \mathrm{~s}^{-1}$. Combined with the low residence time of $33 \mathrm{~ms}$, these shear rates would not be expected to provide sufficient work $\left(\approx 10^{5}\right.$ $\mathrm{Pa})^{45}$ for shear-induced nucleation of the silk molecules.

Silk nanoparticles were reproducibly and reliably manufactured at a $6 \mathrm{~mL}$ scale when key processing parameters were set at levels for optimal nanoparticle properties, as determined in previous work. $^{13,20,32}$ As unreliable nanoparticle manufacture has been implemented in the reduced efficacy of the generic Doxil formulation, LipoDox, ${ }^{41,46}$ one might speculate that increasing the reproducibility of silk nanoparticle manufacture will ultimately lead to better in vitro and in vivo therapeutic profiling. For example, across 16 repeats using three aqueous silk batches over 3 days, silk nanoparticles produced at a 5.5 $\mathrm{cm}$ initial addition height and without stirring had an average size of $131 \pm 7 \mathrm{~nm}$ and a low polydispersity of $0.11 \pm 0.02$. The polydispersity was similar to previously reported values obtained using the same silk concentration and silk-toantisolvent volumetric ratio in manual and microfluidicassisted methodology. ${ }^{8,13,20,32}$ Although the nanoparticle sizes were larger than the literature values $(\approx 100-115 \mathrm{~nm}), 8,13,20,32$ they lie within the optimal size range $(100-200 \mathrm{~nm})$ for drug delivery vehicles. ${ }^{47}$ The nanoparticles were obtained in an average yield of $18 \pm 3 \%$, comparing favorably with previous reports in manual and microfluidic formats. ${ }^{13,32}$ The zeta potential of $-30 \pm 2 \mathrm{mV}$ was higher than previously reported values obtained with manual silk addition. ${ }^{8,20}$ This phenomenon was also observed for microfluidic-assisted manufacture $^{20,32}$ and probably reflects different silk molecule packing arrangements resulting from varying fluid dynamics due to the different flask geometries between the studies.

Silk nanoparticles were highly crystalline, featuring a high $\beta$ sheet content of $56 \pm 1 \%$ and a spectral correlation coefficient of $0.27 \pm 0.03$ over the amide I region, in agreement with previous studies. $^{20,32}$ Increasing the initial addition height to $7.5 \mathrm{~cm}$ resulted in higher droplet impact velocity and kinetic energy, thereby causing larger disturbances in the antisolvent and facilitating mass transfer and solvent shifting. Surprisingly, this change did not significantly affect nanoparticle physicochemical properties, although it resulted in significantly increased yield.

The inverse relationship between nanoparticle size and formulation stirring rate has been observed for globular protein nanoparticles $^{48}$ and polymer emulsions. ${ }^{49}$ As a nanoprecipitation process, aqueous silk desolvation is fundamentally a diffusion-limited solvent shift of water molecules from the silk hydration shell and their replacement with isopropanol molecules. Therefore, mixing efficiency is an important factor that dictates nanoprecipitation outcomes, and magnetic stirring increases the control of macro- to micromixing rates. ${ }^{28}$ In labscale semi-batch manufacture, the silk nanoparticle size and yield showed inverse dependence on the stirring rate, through 0-400 rpm (Figure 2). While stirring has been used for desolvation of regenerated silk obtained from Antheraea mylitta $^{50}$ we believe this is the first report highlighting the importance of stirring rate in a semi-batch system on the outcome of silk fibroin desolvation. When manufactured at 400 rpm stirring rate and $6 \mathrm{~mL}$ scale, the nanoparticle size compared well with literature values for manual semi-batch manufacture, which is typically conducted between 40 and 50
$\mathrm{mL}$ scales without stirring. ${ }^{8,20}$ It is likely that reducing the mixing time by active stirring will result in increased reproducibility of nanoparticle physicochemical properties during scale-up, although experimental proof is needed.

Silk nanoprecipitation occurs during mixing with an antisolvent in which the solubility of at least one type of hydrophilic block is low, and this results in particle nucleation upon supersaturation (i.e., when the silk concentration exceeds the equilibrium solubility). ${ }^{25,51}$ Nucleation follows a minimum Gibbs free-energy self-assembly process via protein-protein association until a critical nucleus size is reached. ${ }^{51}$ This is then followed by particle growth and protein conformational changes for induced fit (Figure 3a). ${ }^{51}$ For most cases, the general mechanism of protein-protein association is defined by at least three steps. ${ }^{51}$ The initial steps are diffusion-limited and occur following complete solvent-antisolvent mixing. First, a random collision of proteins produces a nonspecific encounter complex, which minimizes repulsive long-range electrostatics. ${ }^{51}$ This short-lived complex can then go on to form nuclei with favorable intermolecular interactions, although no change in secondary structure occurs. This process is enthalpy-driven: the establishment of new shortrange attractive forces between silk molecules offsets the entropic loss upon incorporation into the nuclei.

The consequent reduction in protein concentration reduces the rate of further nucleation and, for nuclei exceeding the critical size, leads to growth by thermodynamically controlled stepwise or aggregative mechanisms. ${ }^{27,52}$ The final step involves a structural change between the favorable growth intermediates and the nanoparticle-bound state to maximize attractive intra- and intermolecular interactions. In the case of silk fibroin, this involves conversion of random coil and $\alpha$-helix content to $\beta$-sheet structure. ${ }^{1,32}$ Across all semi-batch formulations, as in previous microfluidic-assisted work, ${ }^{20,32}$ the mixing efficiency correlated with nanoparticle size, while the secondary structure content and thermal stability of silk nanoparticles remained consistent. Hence, nanoprecipitation can be assumed to occur via diffusion-controlled association in the regime where $k_{\mathrm{R} \rightarrow \beta+} \gg k_{1}^{-}$(Figure $3 \mathrm{a}$ ), ${ }^{51}$ so $\beta$-sheet formation occurs at a faster rate than silk molecule diffusion. Applying this assumption to silk fibroin desolvation, the high turbulence created by increasing the stirring rate would increase the meso- and micromixing rates, thereby reducing the total mixing time. ${ }^{27,53}$ Consequently, nucleation rates will increase, causing a fast reduction in supersaturation and arresting further nucleation, thereby resulting in a growth phase with greater homogeneity. The reduction in local silk concentration with lower mixing times also disfavors surfacecontrolled growth processes of nuclei and lowers the chance of successful diffusion-limited collisions of silk molecules with nuclei ${ }^{54}$ prior to structural rearrangement.

Alternatively, as the stirring rate and mixing efficiency increased, solvent shifting from hydrated silk pockets was likely improved prior to $\beta$-sheet enrichment. This would result in tighter packing of the internal nanoparticle architecture. However, variation in the stirring rate caused no significant difference in water content or desorption enthalpy by simultaneous thermal analysis. Furthermore, as water absorption occurs predominantly in the amorphous regions, ${ }^{55}$ variations in secondary structure would be expected. Nevertheless, $\beta$-sheet crystalline content did not vary significantly between nanoparticle formulations, as measured by amide I band deconvolution. ${ }^{32,33,36}$ The elevated decomposition 
temperatures, absence of crystallization exotherms, and shallower glass-transition profiles of nanoparticles compared to freeze-dried silk (Figure 4) reinforced the high nanoparticle crystallinity determined by FTIR. ${ }^{35,56}$ Simultaneous thermal analysis also showed no significant differences between the thermal stabilities of nanoparticles manufactured with different stirring rates. Macromolecule thermal stability is dependent on molecular weight and length, ${ }^{56}$ so this finding indicated that silk molecules incorporated into nanoparticles from all formulations were of a similar weight and length distribution, again reinforcing previous work. ${ }^{20}$

We speculate that the reduction in nanoparticle yield with stirring is due to several factors, including silk film formation on flask walls and insufficient $g$-force for the sedimentation of smaller nanoparticles during centrifugation. Stirring at $400 \mathrm{rpm}$ resulted in the poorest reproducibility of size and yield, which may indicate that hydrodynamic confounders caused by slight differences in feed point position were introduced between experiments. Surprisingly, no significant differences were observed for polydispersity with stirring, suggesting that, in the static system, size distribution was controlled by diffusionlimited or polynuclear surface-controlled growth. ${ }^{54}$ The moderate polydispersity of all formulations arose, in part, due to the time dependence of antisolvent composition, the local regions of high supersaturation at the droplet-antisolvent interface, and the nonuniform nucleation inherent in the semibatch process. Although particle size was affected, the zeta potential remained at a constant level as stirring rate was varied, suggesting that packing geometries were affected by active stirring although the secondary structure content remained consistent.

Particle growth is a thermodynamically driven process and primarily occurs via three diffusion-limited mechanisms: stepwise growth ${ }^{52}$ proceeding through molecular adsorption until the equilibrium silk saturation concentration is reached; Ostwald ripening, ${ }^{57}$ whereby the dissolution of small particles results in the growth of larger particles; and aggregation proceeding according to Smoluchowski kinetics. ${ }^{27}$ The rate of silk nanoparticle growth was also investigated over $24 \mathrm{~h}$ by varying the standing time in the mother liquor before purification. No significant differences in nanoparticle physicochemical properties, yield (Figure 2), or secondary structure content (Figure 3c) were observed for standing times between 0 and $24 \mathrm{~h}$.

The isoelectric point of crystalline silk fibroin lies between $\mathrm{pH} 4$ and 5. ${ }^{58}$ Therefore, silk nanoparticles and silk molecules in the mother liquor have a net negative surface charge. This results in repulsive long-range electrostatic interactions between nanoparticles, providing a high-energy barrier for aggregation and agglomeration and conferring colloidal stability. These repulsive interactions exist between nanoparticles, precursor silk molecules, and newly formed silk nuclei. Hence, once silk nanoparticles reach a key size, they no longer act as templates for stepwise growth, as the repulsive energy barrier and entropic loss are no longer offset by the establishment of favorable short-range bonds and reduction in surface energy. The conversion of amorphous content to $\beta$ sheet structure upon nanoprecipitation can also be considered irreversible at room temperature, in the absence of chaotropic agents. ${ }^{20}$ The tightly bound crystalline architecture, poor solubility of the silk hydrophilic blocks in the mother liquor, and the low polydispersity of nanoparticle size consequently result in unfavorable nanoparticle growth via Ostwald ripening.
This means that screening of operating conditions for nanoparticle manufacture at room temperature can be conducted with maximum time efficiency, increasing throughput.

For example, at $6 \mathrm{~mL}$ scale and $0 \mathrm{~h}$ standing time, the nanoparticle production rate was estimated as $0.41 \mathrm{~g} / \mathrm{h}$ using semi-automated silk dispensing and $0.12 \mathrm{~g} / \mathrm{h}$ using manual silk addition, assuming $23 \%$ nanoparticle yields. ${ }^{20}$ The former value assumed the use of one syringe pump equipped with two syringes, while both processes require an operator intensive setup time of $1 \mathrm{~min}$. Based on this, the time taken to prepare nanoparticle batches for conducting a clinically relevant in vivo study with five rats was calculated. Assuming a nanoparticle blood concentration ${ }^{7}$ of $250 \mu \mathrm{g} \mathrm{mL}^{-1}$ and a rat blood volume of $25.6 \mathrm{~mL}, 6.4 \mathrm{mg}$ of silk nanoparticles would be required per rat. The time taken to obtain the total required mass of $32 \mathrm{mg}$ is 0.08 and $0.26 \mathrm{~h}$ using the semi-automated and manual setups, respectively. However, the total production rate is lowered by the $6 \mathrm{~h}$ purification process, assuming the use of one eight-place centrifuge. Nevertheless, the total production rate can be increased by a syringe pump platform and centrifuge parallelization.

The characterization of the effect of aging on nanoparticle physicochemical properties is important for maximizing shelf life and preventing undesired complications. For this reason, we also examined the long-term stability of silk nanoparticles from all formulations in aqueous conditions for over 1 month at $4{ }^{\circ} \mathrm{C}$ to assess storage capabilities (Figure 5). Similar to previous studies, ${ }^{13,20,32}$ the zeta potential of nanoparticles from all formulations on the day of manufacture was lower than -25 $\mathrm{mV}$, indicating sufficient electrostatic repulsion between particles for moderate aqueous stability. Indeed, silk nanoparticles manufactured across all stirring rates and standing times showed size stability over the entire study period. Some fluctuations in polydispersity and zeta potential occurred for nanoparticles produced from some formulations and, while these changes were significant, they did not follow a trend indicative of time-dependent flocculation, coagulation, or dissolution. $^{32}$ This observation was reinforced by morphological assessments conducted over the time course by SEM, which showed spherical granules without apparent agglomeration or adhesion (Figure 6). The sizes of the freeze-dried particles imaged by SEM were relatively small compared to the $Z$-average size measured using DLS, probably due to the removal of the solvation sphere and bound water during freezedrying. ${ }^{50}$

\section{CONCLUSIONS}

The use of a semi-automated liquid dispensing setup provided consistent, standardized, and higher-throughput manufacture of silk nanoparticles via drop-by-drop desolvation in a semibatch format. Operational parameters investigated for their effect on nanoparticle formation indicated that decreasing the initial addition height from 7.5 to $5.5 \mathrm{~cm}$ reduced the nanoparticle yield. The stirring rate was also a key process parameter that affected silk nanoparticle size, yield, and experiment reproducibility, as stirring at $400 \mathrm{rpm}$ provided the smallest nanoparticle size and the lowest yield of silk nanoparticles. Nanoparticles from all formulations displayed spherical morphologies and showed stability of size and polydispersity for over 1 month when stored as aqueous suspensions at $4{ }^{\circ} \mathrm{C}$. The standing time of silk nanoparticles in the mother liquor was also not a key process parameter, and 
time-efficient manufacture can be achieved at room temperature.

\section{ASSOCIATED CONTENT}

\section{SI Supporting Information}

The Supporting Information is available free of charge at https://pubs.acs.org/doi/10.1021/acsbiomaterials.0c01028.

Exemplary smoothed second-derivative FTIR spectra and peak fitting in the amide I region for nanoparticles, films, and powders (PDF)

\section{AUTHOR INFORMATION}

\section{Corresponding Author}

F. Philipp Seib - Strathclyde Institute of Pharmacy and Biomedical Sciences, University of Strathclyde, Glasgow G4 ORE, U.K.; EPSRC Future Manufacturing Research Hub for Continuous Manufacturing and Advanced Crystallisation (CMAC), University of Strathclyde, Technology and Innovation Centre, Glasgow G1 1RD, U.K.; Leibniz Institute of Polymer Research Dresden, Max Bergmann Center of Biomaterials Dresden, 01069 Dresden, Germany; 이이. orcid.org/0000-00021955-1975; Phone: +44 (0) 141548 2510;

Email: philipp.seib@strath.ac.uk

\section{Authors}

Saphia A. L. Matthew - Strathclyde Institute of Pharmacy and Biomedical Sciences, University of Strathclyde, Glasgow G4 ORE, U.K.

John D. Totten - Strathclyde Institute of Pharmacy and Biomedical Sciences, University of Strathclyde, Glasgow G4 ORE, U.K.; EPSRC Future Manufacturing Research Hub for Continuous Manufacturing and Advanced Crystallisation (CMAC), University of Strathclyde, Technology and Innovation Centre, Glasgow G1 1RD, U.K.

Suttinee Phuagkhaopong - Strathclyde Institute of Pharmacy and Biomedical Sciences, University of Strathclyde, Glasgow G4 ORE, U.K.

Gemma Egan - Strathclyde Institute of Pharmacy and Biomedical Sciences, University of Strathclyde, Glasgow G4 ORE, U.K.

Kimia Witte - Strathclyde Institute of Pharmacy and Biomedical Sciences, University of Strathclyde, Glasgow G4 ORE, U.K.

Yvonne Perrie - Strathclyde Institute of Pharmacy and Biomedical Sciences, University of Strathclyde, Glasgow G4 ORE, U.K.; ๑ orcid.org/0000-0001-8497-2541

Complete contact information is available at:

https://pubs.acs.org/10.1021/acsbiomaterials.0c01028

\section{Author Contributions}

S.A.L.M. designed, collected, analyzed, and interpreted the data and generated the manuscript draft. J.D.T., S.P., G.E., K.W., Y.P., and F.P.S. provided training, advised on experimental design, and contributed to the interpretation of the results. All authors discussed the results and/or provided advice on the experimental analysis. F.P.S. supervised the project and content-edited the manuscript.

\section{Funding}

S.A.L.M. is supported by a Medical Research Scotland Ph.D. Studentship (PhD-1292-2018). J.D.T. is supported by an EPSRC Doctoral Prize Award (EP/R513349/1). S.P. received a fellowship support from the Development and Promotion of Science and Technology Talents Project under the Royal
Government of Thailand Scholarship. G.E. is supported by an EPSRC Doctoral Training in Medical Devices and Health Technologies Ph.D. Studentship (EP/LO15595/1).

\section{Notes}

The authors declare no competing financial interest. All data supporting this research are openly available from https://doi.org/10.15129/5731067a-a6a0-4ea1-bb3aed3eaa89368d.

\section{ACKNOWLEDGMENTS}

The authors thank Dr Alice Turner, Dr Deborah Bowering, Dr Maider Olasolo, and Dr John Parkinson (University of Strathclyde, Scotland, U.K.) for providing training and technical advice. The authors acknowledge that work was carried out in part at the EPSRC Future Manufacturing Research Hub for Continuous Manufacturing and Advanced Crystallization (CMAC) (EP/P006965/1) and supported by a U.K. Research Partnership Fund award from the Higher Education Funding Council for England (Grant HH13054). The authors acknowledge that the electron scanning microscopy work was carried out at the Advanced Materials Research Laboratory, housed within the University of Strathclyde.

\section{ABBREVIATIONS}

Aq., aqueous; DSC, dynamic scanning calorimetry; PDI, polydispersity index; SEM, scanning electron microscopy; TGA, thermogravimetric analysis

\section{REFERENCES}

(1) Holland, C.; Numata, K.; Rnjak-Kovacina, J.; Seib, F. P. The Biomedical Use of Silk: Past, Present, Future. Adv. Healthcare Mater. 2019, 8, No. 1800465

(2) Janani, G.; Kumar, M.; Chouhan, D.; Moses, J. C.; Gangrade, A.; Bhattacharjee, S.; Mandal, B. B. Insight into Silk-Based Biomaterials: From Physicochemical Attributes to Recent Biomedical Applications. ACS Appl. Bio Mater. 2019, 2, 5460-5491.

(3) Wenk, E.; Merkle, H. P.; Meinel, L. Silk Fibroin as a Vehicle for Drug Delivery Applications. J. Controlled Release 2011, 150, 128-141.

(4) Mehrotra, S.; Chouhan, D.; Konwarh, R.; Kumar, M.; Jadi, P. K.; Mandal, B. B. Comprehensive Review on Silk at Nanoscale for Regenerative Medicine and Allied Applications. ACS Biomater. Sci. Eng. 2019, 5, 2054-2078.

(5) Chouhan, D.; Mandal, B. B. Silk Biomaterials in Wound Healing and Skin Regeneration Therapeutics: From Bench to Bedside. Acta Biomater. 2020, 103, 24-51.

(6) Seib, F. P. Silk Nanoparticles-an Emerging Anticancer Nanomedicine. AIMS Bioeng. 2017, 4, 239-258.

(7) Maitz, M. F.; Sperling, C.; Wongpinyochit, T.; Herklotz, M.; Werner, C.; Seib, F. P.; et al. Biocompatibility Assessment of Silk Nanoparticles: Hemocompatibility and Internalization by Human Blood Cells. Nanomedicine 2017, 12, 2633-2642.

(8) Wongpinyochit, T.; Uhlmann, P.; Urquhart, A. J.; Seib, F. P. PEGylated Silk Nanoparticles for Anticancer Drug Delivery. Biomacromolecules 2015, 16, 3712-3722.

(9) Sofregen. Sofregen Receives $510(\mathrm{k})$ Clearance for Silk Voice https://www.sofregen.com/press-release-3/press-release-3 (accessed May 1, 2020).

(10) Xiao, L.; Lu, G.; Lu, Q.; Kaplan, D. L. Direct Formation of Silk Nanoparticles for Drug Delivery. ACS Biomater. Sci. Eng. 2016, 2, 2050-2057.

(11) Xu, Z.; Shi, L.; Yang, M.; Zhu, L. Preparation and Biomedical Applications of Silk Fibroin-Nanoparticles Composites with Enhanced Properties - A Review. Mater. Sci. Eng., C 2019, 95, 302-311. 
(12) Konwarh, R. Can the Venerated Silk Be the Next-Generation Nanobiomaterial for Biomedical-Device Designing, Regenerative Medicine and Drug Delivery? Prospects and Hitches. Bio-Des. Manuf. 2019, 2, 278-286.

(13) Wongpinyochit, T.; Johnston, B. F.; Seib, F. P. Manufacture and Drug Delivery Applications of Silk Nanoparticles. J. Visualized Exp. 2016, No. e54669.

(14) Seib, F. P.; Jones, G. T.; Rnjak-Kovacina, J.; Lin, Y.; Kaplan, D. L. PH-Dependent Anticancer Drug Release from Silk Nanoparticles. Adv. Healthcare Mater. 2013, 2, 1606-1611.

(15) Yang, P.; Dong, Y.; Huang, D.; Zhu, C.; Liu, H.; Pan, X.; Wu, C. Silk Fibroin Nanoparticles for Enhanced Bio-Macromolecule Delivery to the Retina. Pharm. Dev. Technol. 2019, 24, 575-583.

(16) Totten, J. D.; Wongpinyochit, T.; Seib, F. P. Silk Nanoparticles: Proof of Lysosomotropic Anticancer Drug Delivery at Single-Cell Resolution. J. Drug Targeting 2017, 25, 865-872.

(17) Wongpinyochit, T.; Johnston, B. F.; Seib, F. P. Degradation Behavior of Silk Nanoparticles-Enzyme Responsiveness. ACS Biomater. Sci. Eng. 2018, 4, 942-951.

(18) Crivelli, B.; Perteghella, S.; Bari, E.; Sorrenti, M.; Tripodo, G.; Chlapanidas, T.; Torre, M. L. Silk Nanoparticles: From Inert Supports to Bioactive Natural Carriers for Drug Delivery. Soft Matter 2018, 14, 546-557.

(19) Gupta, V.; Aseh, A.; Ríos, C. N.; Aggarwal, B. B.; Mathur, A. B. Fabrication and Characterization of Silk Fibroin-Derived Curcumin Nanoparticles for Cancer Therapy. Int. J. Nanomed. 2009, 4, 115122.

(20) Solomun, J. I.; Totten, J. D.; Wongpinyochit, T.; Florence, A. J.; Seib, F. P. Manual versus Microfluidic-Assisted Nanoparticle Manufacture: Impact of Silk Fibroin Stock on Nanoparticle Characteristics. ACS Biomater. Sci. Eng. 2020, 6, 2796-2804.

(21) Zhao, Z.; Li, Y.; Xie, M.-B. Silk Fibroin-Based Nanoparticles for Drug Delivery. Int. J. Mol. Sci. 2015, 16, 4880-4903.

(22) Gholami, A.; Tavanai, H.; Moradi, A. R. Production of Fibroin Nanopowder through Electrospraying. J. Nanopart. Res. 2011, 13, 2089-2098

(23) Toprakcioglu, Z.; Challa, P. K.; Morse, D. B.; Knowles, T. Attoliter Protein Nanogels from Droplet Nanofluidics for Intracellular Delivery. Sci. $A d v$. 2020, 6, No. eaay7952.

(24) Lozano-Pérez, A. A.; Montalbán, M. G.; Aznar-Cervantes, S. D.; Cragnolini, F.; Cenis, J. L.; Víllora, G. Production of Silk Fibroin Nanoparticles Using Ionic Liquids and High-Power Ultrasounds. J. Appl. Polym. Sci. 2015, 132, No. 41702.

(25) Tarhini, M.; Greige-Gerges, H.; Elaissari, A. Protein-Based Nanoparticles: From Preparation to Encapsulation of Active Molecules. Int. J. Pharm. 2017, 522, 172-197.

(26) Vauthier, C.; Bouchemal, K. Methods for the Preparation and Manufacture of Polymeric Nanoparticles. Pharm. Res. 2009, 26, $1025-1058$.

(27) Botet, R.; Roger, K. How Do Interactions Control Droplet Size during Nanoprecipitation? Curr. Opin. Colloid Interface Sci. 2016, 22, $108-112$.

(28) Baldyga, J.; Pohorecki, R. Turbulent Micromixing in Chemical Reactors - a Review. Chem. Eng. J. 1995, 58, 183-195.

(29) Nisal, A.; Kalelkar, C.; Bellare, J.; Lele, A. Rheology and Microstructural Studies of Regenerated Silk Fibroin Solutions. Rheol. Acta 2013, 52, 833-840.

(30) Ianovska, M. A.; Mulder, P. P. M. F. A.; Verpoorte, E. Development of Small-Volume, Microfluidic Chaotic Mixers for Future Application in Two-Dimensional Liquid Chromatography. RSC Adv. 2017, 7, 9090-9099.

(31) Rode García, T.; García, Ac. A.; Lalloz, A.; Lacasse, F. X.; Hildgen, P.; Rabanel, J. M.; Banquy, X. Unified Scaling of the Structure and Loading of Nanoparticles Formed by Diffusion-Limited Coalescence. Langmuir 2018, 34, 5772-5780.

(32) Wongpinyochit, T.; Totten, J. D.; Johnston, B. F.; Seib, F. P. Microfluidic-Assisted Silk Nanoparticle Tuning. Nanoscale Adv. 2019, $1,873-883$
(33) Yang, H.; Yang, S.; Kong, J.; Dong, A.; Yu, S. Obtaining Information about Protein Secondary Structures in Aqueous Solution Using Fourier Transform IR Spectroscopy. Nat. Protoc. 2015, 10, 382-396.

(34) Shimanovich, U.; Ruggeri, F. S.; De Genst, E.; Adamcik, J.; Barros, T. P.; Porter, D.; Müller, T.; Mezzenga, R.; Dobson, C. M.; Vollrath, F.; Holland, C.; Knowles, T. P. J. Silk Micrococoons for Protein Stabilisation and Molecular Encapsulation. Nat. Commun. 2017, 8, No. 15902.

(35) Hu, X.; Kaplan, D.; Cebe, P. Determining Beta-Sheet Crystallinity in Fibrous Proteins by Thermal Analysis and Infrared Spectroscopy. Macromolecules 2006, 39, 6161-6170.

(36) Griebenow, K.; Santos, A. M.; Carrasquillo, K. G. Secondary Structure of Proteins in the Amorphous Dehydrated State Probed by FTIR Spectroscopy. Dehydration-Induced Structural Changes and Their Prevention. Internet J. Vib. Spectrosc. 1999, 3, 1-2.

(37) Riesen, R.; Vogel, K.; Schubnell, M. DSC by the TGA/ SDTA851e Considering Mass Changes. J. Therm. Anal. Calorim. 2001, 64, 243-252.

(38) Thermal Analysis of Polymers. Fundamentals and Applications; Menczel, J. D.; Prime, R. B., Eds.; Wiley: Hoboken, New Jersey, 2009.

(39) Mottaghitalab, F.; Farokhi, M.; Shokrgozar, M. A.; Atyabi, F.; Hosseinkhani, H. Silk Fibroin Nanoparticle as a Novel Drug Delivery System. J. Controlled Release 2015, 206, 161-176.

(40) Sindhwani, S.; Syed, A. M.; Ngai, J.; Kingston, B. R.; Maiorino, L.; Rothschild, J.; MacMillan, P.; Zhang, Y.; Rajesh, N. U.; Hoang, T.; Wu, J. L. Y.; Wilhelm, S.; Zilman, A.; Gadde, S.; Sulaiman, A.; Ouyang, B.; Lin, Z.; Wang, L.; Egeblad, M.; Chan, W. C. W. The Entry of Nanoparticles into Solid Tumours. Nat. Mater. 2020, 19, $566-575$

(41) Agrahari, V.; Agrahari, V. Facilitating the Translation of Nanomedicines to a Clinical Product: Challenges and Opportunities. Drug Discovery Today 2018, 23, 974-991.

(42) Muthu, M. S.; Wilson, B. Challenges Posed by the Scale-up of Nanomedicines. Nanomedicine 2012, 7, 307-309.

(43) Pham, D. T.; Saelim, N.; Tiyaboonchai, W. Design of Experiments Model for the Optimization of Silk Fibroin Based Nanoparticles. Int. J. Appl. Pharm. 2018, 10, 195-201.

(44) Zhang, Y.-Q.; Shen, W.-D.; Xiang, R.-L.; Zhuge, L.-J.; Gao, W.J.; Wang, W.-B. Formation of Silk Fibroin Nanoparticles in WaterMiscible Organic Solvent and Their Characterization. J. Nanopart. Res. 2007, 9, 885-900.

(45) Dunderdale, G. J.; Davidson, S. J.; Ryan, A. J.; Mykhaylyk, O. O. Flow-Induced Crystallisation of Polymers from Aqueous Solution. Nat. Commun. 2020, 11, No. 3372.

(46) Anselmo, A. C.; Mitragotri, S. Nanoparticles in the Clinic. Bioeng. Transl. Med. 2016, 1, 10-29.

(47) Wacker, M. Nanocarriers for Intravenous Injection - The Long Hard Road to the Market. Int. J. Pharm. 2013, 457, 50-62.

(48) Rahimnejad, M.; Najafpour, G.; Bakeri, G. Investigation and Modeling Effective Parameters Influencing the Size of BSA Protein Nanoparticles as Colloidal Carrier. Colloids Surf., A 2012, 412, 96100.

(49) Crucho, C. I. C.; Barros, M. T. Polymeric Nanoparticles: A Study on the Preparation Variables and Characterization Methods. Mater. Sci. Eng., C 2017, 80, 771-784.

(50) Kundu, J.; Chung, Y.-I.; Kim, Y. H.; Tae, G.; Kundu, S. C. Silk Fibroin Nanoparticles for Cellular Uptake and Control Release. Int. J. Pharm. 2010, 388, 242-250.

(51) Camacho, C. J.; Kimura, S. R.; DeLisi, C.; Vajda, S. Kinetics of Desolvation-Mediated Protein-Protein Binding. Biophys. J. 2000, 78, 1094-1105.

(52) You, H.; Fang, J. Particle-Mediated Nucleation and Growth of Solution-Synthesized Metal Nanocrystals: A New Story beyond the LaMer Curve. Nano Today 2016, 11, 145-167.

(53) Galisteo-González, F.; Molina-Bolívar, J. A. Systematic Study on the Preparation of BSA Nanoparticles. Colloids Surf., B 2014, 123, 286-292. 
(54) Gao, G.; Wang, Y.; Cao, G.; Wang, Y. Nanostructures and Nanomaterials Synthesis, Properties, and Applications, 2nd ed.; World Scientific: Singapore, 2011.

(55) Motta, A.; Fambri, L.; Migliaresi, C. Regenerated Silk Fibroin Films: Thermal and Dynamic Mechanical Analysis. Macromol. Chem. Phys. 2002, 203, 1658-1665.

(56) Um, I. C.; Kweon, H. Y.; Park, Y. H.; Hudson, S. Structural Characteristics and Properties of the Regenerated Silk Fibroin Prepared from Formic Acid. Int. J. Biol. Macromol. 2001, 29, 91-97. (57) Thanh, N. T. K.; Maclean, N.; Mahiddine, S. Mechanisms of Nucleation and Growth of Nanoparticles in Solution. Chem. Rev. 2014, 114, 7610-7630.

(58) Seib, F. P.; Maitz, M. F.; Hu, X.; Werner, C.; Kaplan, D. L. Impact of Processing Parameters on the Haemocompatibility of Bombyx mori Silk Films. Biomaterials 2012, 33, 1017-1023. 